\title{
Trombólisis en niños con tromboembolia pulmonar asociada a COVID-19
}

\section{Thrombolysis in children with COVID-19-associated pulmonary embolism}

\author{
Mateo Porres-Aguilar, ${ }^{1 *}$ Adriana Torres-Machorro² y Moisés Aurón ${ }^{3}$ \\ ${ }^{1}$ McGill University, Jewish General Hospital, Centre of Excellence in Thrombosis and Anticoagulation Care, División de Medicina de Trombosis, \\ Departamento de Medicina, Montreal, Quebec, Canadá; 'Instituto Nacional de Cardiología "Ignacio Chávez", Departamento de Cirugía Vascular y \\ Terapia Endovascular, Ciudad de México, México; ${ }^{3}$ Cleveland Clinic, Pediatrics Institute, Departamento de Medicina Hospitalaria de Adultos y \\ Pediatría, Cleveland, Ohio, Estados Unidos
}

Hemos leído con interés el artículo de Ávila-Castro y colaboradores. ${ }^{1}$ Actualmente existe poca evidencia en cuanto al papel de la trombólisis sistémica y la trombólisis guiada por catéter en niños con tromboembolia pulmonar severa. ${ }^{2}$ Dicho lo anterior, quisiéramos expresar y compartir las siguientes interrogantes: ¿Cuáles son los regímenes terapéuticos de trombólisis sistémica utilizados en los hospitales de los autores? En caso de que hagan uso de la trombólisis sistémica en dicho contexto clínico, ¿prefieren utilizar un régimen de dosis baja o alta? ¿Cuál es la frecuencia de la hemorragia mayor e intracraneal en los niños?

Recientemente se han publicado series de casos y análisis retrospectivos sobre el uso de trombólisis guiada por catéter en tromboembolia pulmonar pediátrica, que demostraron una eficacia aceptable y menor riesgo de hemorragia mayor, incluyendo la intracraneal. ${ }^{3}$ ¿Creen los autores en el potencial beneficio dentro de las estrategias terapéuticas de reperfusión? No cabe duda de que urgen estudios prospectivos y ensayos clínicos aleatorizados que aborden dichas interrogantes sobre el futuro de la trombólisis en sus diferentes modalidades, sobre todo en el contexto de la pandemia de COVID-19.

\section{Bibliografía}

1. Ávila-Castro D, Ortiz-Torres G, Sánchez-Jara B, Valle-Cárdenas T, Aquino-Fernández E, González-Ávila Al, et al. Propuesta para el manejo de la coagulopatía asociada a COVID-19 en niños. Gac Med Mex 2020;156:344-353.

2. Tarango C, Manco-Jonhson M. Pediatric thrombolysis: a practical approach. Front Pediatr 2017;5:260. DOI: 10.3389/fped.2017.00260.

3. Belsky J, Warren P, Stanek J, Kumar R. Catheter-directed thrombolysis for submassive pulmonary embolism in children: a case series. Pediatr Blood Cancer. 2020;67(4):e28144. DOI: 10.1002/pbc.28144.

\footnotetext{
Correspondencia:

Fecha de recepción: 23-09-2020

*Mateo Porres-Aguilar

Fecha de aceptación: 01-10-2020

E-mail: mporres1980@gmail.com

DOI: 10.24875/GMM.20000701

Gac Med Mex. 2020;156:618

Disponible en PubMed

0016-3813/@ 2020 Academia Nacional de Medicina de México, A.C. Publicado por Permanyer. Este es un artículo open access bajo la licencia CC BY-NC-ND (http://creativecommons.org/licenses/by-nc-nd/4.0/).
} 